\title{
Environmental inversion using high-resolution matched-field processing $^{\text {a) }}$
}

\author{
Cristiano Soares ${ }^{b)}$ and Sérgio M. Jesus \\ Institute for Systems and Robotics, Universidade do Algarve, Campus de Gambelas, PT-8005-139 Faro, \\ Portugal \\ Emanuel Coelho ${ }^{\mathrm{c})}$ \\ NATO Undersea Research Centre, Viale San Bartolomeo 400, I-19138 La Spezia, Italy
}

(Received 5 March 2007; revised 14 September 2007; accepted 17 September 2007)

\begin{abstract}
This paper considers the inversion of experimental field data collected with light receiving systems designed to meet operational requirements. Such operational requirements include system deployment in free drifting configurations and a limited number of acoustic receivers. A well-known consequence of a reduced spatial coverage is a poor sampling of the vertical structure of the acoustic field, leading to a severe ill-conditioning of the inverse problem and data to model cost function with a massive sidelobe structure having many local extrema. This causes difficulties to meta-heuristic global search methods, such as genetic algorithms, to converge to the true model parameters. In order to cope with this difficulty, broadband high-resolution processors are proposed for their ability to significantly attenuate sidelobes, as a contribution for improving convergence. A comparative study on simulated data shows that high-resolution methods did not outperform the conventional Bartlett processor for pinpointing the true environmental parameter when using exhaustive search. However, when a meta-heuristic technique is applied for exploring a large multidimensional search space, high-resolution methods clearly improved convergence, therefore reducing the inherent uncertainty on the final estimate. These findings are supported by the results obtained on experimental field data obtained during the Maritime Rapid Environmental Assessment 2003 sea trial. (C) 2007 Acoustical Society of America. [DOI: 10.1121/1.2799476]
\end{abstract}

PACS number(s): 43.30.Wi, 43.30.Pc, 43.60.Cg, 43.60.Pt [AIT]

Pages: $3391-3404$

\section{INTRODUCTION}

During the 1990s, the problem of simultaneously estimating multiple ocean parameters by means of inversion of acoustic data collected with vertical receiver arrays aroused considerable interest in the underwater acoustic community. Several studies with experimental field data have demonstrated the viability of environmental inversion based on matched-field processing (MFP) with multiple unknown parameters. MFP-based inversion techniques perform a comparison of the full pressure field (amplitude and phase) received at an array of hydrophones with computer generated field replicas, usually by means of a correlation. ${ }^{1,2}$

MFP, originally proposed for source localization, was first formulated by Bucker ${ }^{3}$ as he used realistic environmental models, introduced the concept of ambiguity surface, and demonstrated that there was enough complexity of the wave field to allow inversion. The field complexity can be measured in terms of the number of contributing normal modes, which is directly related to the degree of uniqueness of the inverse problem's solution. The number of contributing normal modes varies with physical parameters such as fre-

\footnotetext{
a) Portions of this work were presented at the European Conference on Underwater Acoustics on June 2006, Carvoeiro, Portugal.

${ }^{b)}$ Electronic mail: csoares@ualg.pt

${ }^{c}$ Current address: Naval Research Laboratory, Code 7322, Bldg. 1009, Room C128, Stennis Space Center, MS 39529.
}

quency and water depth, among others. The idea of using vertical array is to spatially sample that normal modes structure.

Then it was recognized that MFP could be also applied to environmental inversion problems, such as the inversion of the ocean water column ${ }^{4}$ and bottom properties. ${ }^{5-8}$ As, in general, direct inversion of the acoustic field is not possible, the inverse problem is usually posed as a nonlinear optimization problem aiming at the maximization of the match between the measured acoustic field and the replica field calculated for candidate parameter values. In most cases multiple unknown parameters enter the optimization problem, therefore resulting in large search spaces. Thus, exhaustive search is not a viable practice. Gradient methods are also not viable due to the existence of many local extrema. This requires employment of efficient global optimization methods such as genetic algorithms (GA) and simulated anealing (SA).

Collins et al. first proposed including environmental parameters in the search space in the context of range-depth source localization as an attempt to overcome model mismatch. ${ }^{9}$ In this study with synthetic data the optimization was carried out with SA. There have been a number of papers on experimental results on inversion of acoustic data for geometric and environmental parameters ${ }^{10-12}$ aimed at sup- 
porting source localization. Other experimental studies used global optimization methods for the estimation of oceanbottom properties. ${ }^{5,6,13}$

In most of these studies, part of the success of MFPbased inversion techniques is explained by the fact that most acoustic experiments were carried out under highly controlled conditions, employing acoustic reception systems with a large number of receivers, moored arrays, and low frequency acoustic projectors. In other words, acoustic systems traditionally employed are research directed apparatus, bulky and difficult to operate for their deployment requirements, and are therefore not suitable for operational use.

Current developments of receiver systems go in the sense of reducing their overall size along with the length of the array itself and the number of receivers with the objective of reducing the cost and deployment requirements of these systems. The point is that if a sparse array is used then higher order modes are undersampled, i.e., the spatial Nyquist criterion is not taken into account, and MFP cannot effectively take advantage of that field complexity. The result is that the ambiguity surface, or hypersurface in the case of multiple unknown parameters, will show many sidelobes spread over the search space comparable with the main peak at the true solution, leading to a severely ill-conditioned problem with a large number of local extrema. ${ }^{14}$ When dealing with real data, the inherent model mismatch and the presence of noise create a situation where there is no assurance of existence of an optimum solution in coincidence with (or even close to) the true model parameters. An additional concern arises when the optimization problem is solved with aid of metaheuristic methods such as a genetic algorithm (GA) even in the absence of noise and model mismatch. The large number of local extrema associated with the typically large search space is a major difficulty factor to this class of search methods in attaining convergence to the true model parameters.

This problem leads to an important discussion in MF approaches, which is on the ability of the processor to attenuate sidelobes. In the past, much effort has gone into developing processor techniques with increased sidelobe attenuation capabilities. One of the main topics was the debate on incoherent and coherent processors, where it was claimed that using coherent processors would allow for increased sidelobe attenuation in comparison to the incoherent counterparts. ${ }^{15-20}$ Another possibility would be the employment of high-resolution processors. However, this possibility has not been significantly considered in the past due to the generalized notion that those methods nave weak probabilities of successful application with experimental data due to there high sensitivity to model mismatch. This paper proposes broadband and high-resolution MF processors for environmental inversion of acoustic data collected with the Acoustic Oceanographic Buoy (AOB) ${ }^{21,22}$ a light receiving system with a sparse vertical array deployed in a free-drifting configuration, where the signals were transmitted by a towed acoustic source. Here, the application of high-resolution processors to environmental inversion is motivated by their possibility to significantly improve the convergence of global search algorithms due to their increased ability to attenuate sidelobes. Simulation results show that in the case of an ex- haustive search, conventional processing is more capable of correctly pinpointing the maximum at the true parameter value than the proposed high-resolution methods. However, both synthetic and experimental inversion results obtained with a GA show that the high-resolution methods can significantly improve convergence to the global solution of the inverse problem.

This paper is organized as follows: Section II develops broadband and high-resolution matched-field processors; Sec. III presents a synthetic study aimed at understanding the difficulties in applying high-resolution processors and comparing them with the conventional processor; Sec. IV gives a description of the MREA'03 sea trial, and presents experimental results obtained with the proposed matched-field processors; finally, Sec. V draws final conclusions.

\section{MATCHED-FIELD PROCESSORS FOR PARAMETER ESTIMATION}

In order to cope with the difficulty that arises from using a sparse array to collect acoustic data in conjunction with meta-heuristic search methods, the following proposes various broadband (BB) matched-field processors. There are at least two issues that can contribute to alleviate the illconditioning of the inversion problem: One is to efficiently use the spectral components of the acoustic field by exploiting field coherence across the spectral band, which has been claimed in the literature as a means of exploiting additional information contained in the acoustic field; the other is the application of matched-field processors based on a BB data model exploiting that cross-frequency coherence. The following matched-field processors are considered herein: A BB Bartlett processor; ${ }^{2,1}$ a BB minimum-variance (MV) processor; ${ }^{23,2,1}$ and a subspace based method, the BB Multiple Signal Classification (MUSIC) processor. $^{24}$ The MV and the MUSIC processors are high-resolution methods, with an increased ability for attenuating sidelobes in comparison to the Bartlett processor.

\section{A. The broadband data model}

The broadband data model for the acoustic data received at an $L$-receiver array is written as a concatenation of $K$ narrow-band signals $\underline{Y}\left(\omega_{k}\right)$ at discrete frequencies of interest $\omega_{k}$ :

$$
\underline{Y}=\left[\underline{Y}^{T}\left(\omega_{1}\right), \ldots, \underline{Y}^{T}\left(\omega_{k}\right), \ldots, \underline{Y}^{T}\left(\omega_{K}\right)\right]^{T}=\mathbf{H}(\underline{\theta}) \underline{\tilde{S}}+\underline{N}
$$

in order to introduce, as much as possible, a common framework for the narrow-band and broadband cases (see Ref. 20 for a detailed discussion). This data model allows for accounting for the field coherence across frequencies. The vector $\underline{\theta}$ represents the channel parameters and matrix $\mathbf{H}(\underline{\theta})$ is the channel response matrix given as 
$\mathbf{H}(\underline{\theta})=\left[\begin{array}{ccccc}\underline{H}\left(\omega_{1}, \underline{\theta}\right) & \cdots & \underline{0}_{k-1} & \ldots & \underline{0}_{K-2} \\ \underline{0}_{1} & \cdots & \underline{H}\left(\omega_{k}, \underline{\theta}\right) & \ldots & \underline{0}_{1} \\ \underline{0}_{K-2} & \cdots & \underline{0}_{K-k} & \cdots & \underline{H}\left(\omega_{K}, \underline{\theta}\right)\end{array}\right]$,

where the $\underline{H}\left(\omega_{k}, \underline{\theta}\right)$ is an $L$-vector representing the channel response at frequency $\omega_{k}, k=1, \ldots, K .0_{k}$ is a vector with $k L$ zeros. This channel matrix is analogous to that used in classical array processing models for multiple emitters. In the present case, each column is relative to a frequency $\omega_{k}$, however, the channel vectors do not overlap across the columns, in order to keep frequencies separated. The channel matrix has $K L$ rows and $K$ columns. The vector $\underline{\tilde{S}}$ has entries $S\left(\omega_{k}\right) \alpha\left(\omega_{k}\right)$, i.e., the source spectrum multiplied by a random perturbation factor at each frequency $\omega_{k} \in\left[\omega_{1}, \omega_{K}\right]$. The random perturbation factor $\alpha\left(\omega_{k}\right)$ appears as an attempt to account for unmodeled ocean inhomogeneities. ${ }^{20}$ The vector $\underline{N}$ represents the noise, which is assumed Gaussian zero mean, and follows the same notation as $\underline{Y}$ in Eq. (1). Let

$$
\mathbf{C}_{Y Y}=E\left\{\underline{Y Y^{H}}\right\}=\mathbf{H C}_{S S} \mathbf{H}^{H}+\sigma_{N}^{2} \mathbf{I}
$$

be a generic definition of the spectral density matrix (SDM) for $\underline{Y}$ defined in Eq. (1), where $\mathbf{C}_{S S}$ is the signal matrix given by $E\left\{\underline{\tilde{S}}^{H}\right\}$, and $\sigma_{N}^{2}$ the noise variance. The dimensions of the SDM $\mathbf{C}_{Y Y}$ are $K L \times K L$ consisting of $L \times L$ cross-frequency SDMs $\mathbf{C}_{Y Y}\left(\omega_{k 1}, \omega_{k 2}\right)$. The SDMs for $k_{1} \neq k_{2}$ are noiseless according to Eq. (3) since it is assumed that the noise is uncorrelated both across space and frequency. Concerning the signal component, if the signal receptions are fully coherent, then it just happens that $\mathbf{C}_{S S}=\underline{S S}$, which has rank equal one. On the other hand, if the emitted wave form is a random signal, then $\mathbf{C}_{S S}=\operatorname{diag}\left[\sigma_{S}^{2}\left(\omega_{1}\right), \ldots, \sigma_{S}^{2}\left(\omega_{k}\right), \ldots, \sigma_{S}^{2}\left(\omega_{K}\right)\right]$, with $\sigma_{S}^{2}\left(\omega_{k}\right)=\mathrm{E}\left\{\alpha^{*}\left(\omega_{k}\right) \alpha\left(\omega_{k}\right) S^{*}\left(\omega_{k}\right) S\left(\omega_{k}\right)\right\}$. In that case the rank of the signal matrix is equal to $K$. Note that for this case the SDM $\mathbf{C}_{Y Y}$ consists only of block matrices in the diagonal. The intermediate case is that where the rank of the signal matrix can vary between 1 and $K$, representing partial frequency cross correlation. This model is the most generic in the framework of a full broadband data model. At this point we stress the relevance of the rank of the signal matrix: From the signals' point of view, the ocean represents a system with a response that may have features of random nature. In other words, a sequence of deterministic emissions is generally received as a random sequence. The degree of randomness seen at the receivers may depend not only on ocean inhomogeneities such as sea surface roughness, but on small motion of the receivers. There may be a significant contribution related to the variability in the geometry of the experimental setup caused by drifts both of the emitter and the receivers. In terms of the BB data model, it should be noted that the channel response is assumed to be deterministic, but in practice there are both channel random features and parameter variability over the observation window, which is to be accounted for by the introduction of the random perturbation factor. These phenomena may have an impact on the coherence across the spectral band and therefore on the rank of the $\mathbf{C}_{S S}$ signal matrix. Next, the three above-mentioned processors will be derived using the BB data model.

\section{B. The BB Bartlett processor}

Conventional or Bartlett matched-field processors are the most popular in underwater acoustic estimation problems, since they have been used in virtually every study on MFP. The frequency domain Bartlett processor, also called linear processor, performs matched-field beamforming by weighting the output of the array elements at different frequencies and summing over all elements:

$$
P_{B}(\underline{\theta})=\mathrm{E}\left\{\operatorname{tr}\left[\mathbf{w}^{H}(\underline{\theta}) \underline{Y}\left(\underline{\theta}_{0}\right) \underline{Y}^{H}\left(\underline{\theta}_{0}\right) \mathbf{w}(\underline{\theta})\right]\right\},
$$

where $\mathbf{w}$ is a weighting matrix with $K$ columns. Note that it is assumed that the acoustic field is zero mean without loss of generality. Replacing with Eq. (3) and by performing a few ordinary algebra steps to maximize this criterion with respect to $\mathbf{w}(\underline{\theta})$ under the constraint $\operatorname{tr}\left[\mathbf{w}^{H}(\underline{\theta}) \mathbf{w}(\underline{\theta})\right]=1$ the following function is obtained:

$$
P_{\mathrm{B}}(\underline{\theta})=\frac{\operatorname{tr}\left[\mathbf{H}^{H}(\underline{\theta}) \mathbf{C}_{Y Y} \mathbf{H}(\underline{\theta}) \mathbf{C}_{S S}\right]}{\operatorname{tr}\left[\mathbf{H}^{H}(\underline{\theta}) \mathbf{H}(\underline{\theta}) \mathbf{C}_{S S}\right]} .
$$

This is the BB Bartlett processor for generic assumptions on the emitted signal component in terms of the cross-frequency structure. Other functions can be obtained by working out assumptions on $\mathbf{C}_{S S}$ comprehending either uncorrelated or fully correlated frequency components.

\section{The BB minimum-variance processor}

The Bartlett processor generally has important limitations in terms of sidelobe attenuation. This might become a major difficulty in multiparameter estimation problems, when several unknown parameters are considered. As an attempt to alleviate such limitation Capon ${ }^{23}$ proposed a processor commonly known as Minimum Variance Distortionless Response (MVDR) processor. The derivation of the broadband MV processor is well documented in the literature and follows a similar notation as that for the above-presented BB Bartlett processor resulting as

$$
P(\underline{\theta})=\frac{\operatorname{tr}\left[\mathbf{H}^{H}(\underline{\theta}) \mathbf{H}(\underline{\theta}) \mathbf{C}_{S S}\right]}{\operatorname{tr}\left[\mathbf{H}^{H}(\underline{\theta}) \mathbf{C}_{Y Y}^{-1} \mathbf{H}(\underline{\theta}) \mathbf{C}_{S S}\right]} .
$$

With regard to calculations, the MV processor presents the need to invert the SDM $\mathbf{C}_{Y Y}$, which can be done in a straightforward fashion provided that the SDM is of rank $K L$. In practice, this requires the number of snapshots of the received signal $Y$ to be equal or larger than $K L$ for calculating the sample SDM. Otherwise, it may be necessary to diagonal overload the SDM, as suggested in Ref. 25.

\section{The BB MUSIC processor}

The BB data model has been discussed in Sec. II A in the context of channel variability and ocean inhomogeneities, raising the question of the rank of the signal matrix $\mathbf{C}_{S S}$, which is equivalent to the signal subspace dimension. Historically, the subspace approach has been reported in the framework of classical beamforming for direction-of-arrival estimation and detection of emitters where the signal subspace dimension is the number of independent emitters detected. The present case consists of a single emitter radiating 
at several frequencies. In this context the dimension of the signal subspace is related to the degree of spectral coherence of the acoustic field at the discrete frequencies of interesthence a measure of the cross correlation of those spectral components, while the number of frequencies considered is always known. In general the SDM defined in Eq. (3) can be expressed in terms of the eigendecomposition

$$
\mathbf{C}_{Y Y}=\mathbf{U}_{S} \boldsymbol{\Lambda}_{S} \mathbf{U}_{S}^{H}+\sigma_{N}^{2} \mathbf{U}_{N} \mathbf{U}_{N}^{H},
$$

where the data space is separated into signal and noise subspaces. This is an ordinary eigenfactorization with the fact that the eigenvalues and eigenvectors appear separated, with the subscripts $S$ and $N$ denoting signal subspace and noise subspace, respectively.

The idea would be to hypothesize a geometric solution for the eigenproblem, in particular, concerning the signal subspace represented by $\boldsymbol{\Lambda}_{S}$ and $\mathbf{U}_{S}$. However, since the data model assumes an arbitrary signal subspace dimension varying from 1 to $K$, the best that can be asserted is that the span of the signal subspace is the same as that of the columns of

$$
\frac{\mathbf{H}\left(\underline{\theta}_{0}\right) \mathbf{C}_{S S}^{1 / 2}}{\sqrt{\operatorname{tr}\left[\mathbf{H}\left(\underline{\theta}_{0}\right) \mathbf{C}_{S S} \mathbf{H}^{H}\left(\underline{\theta}_{0}\right)\right]}} .
$$

The dimension of the signal subspace is equal to the rank of $\mathbf{C}_{S S}$. This defines the signal subspace in agreement with Eq. (3), but according to $\mathrm{Schmidt}^{24}$ the signal subspace can also be defined by its orthogonal complement-the noise subspace. This is acceptable due to the orthogonality between the columns of $\mathbf{U}_{S}$ and $\mathbf{U}_{N}$ in Eq. (7), i.e., $\mathbf{U}_{S} \mathbf{U}_{N}=\mathbf{0}$. Thus, as the span of $\mathbf{U}_{S}$ is that of Eq. (8), the condition

$$
\mathbf{U}_{N}^{H} \frac{\mathbf{H}\left(\underline{\theta}_{0}\right) \mathbf{C}_{S S}^{1 / 2}}{\sqrt{\operatorname{tr}\left[\mathbf{H}\left(\underline{\theta}_{0}\right) \mathbf{C}_{S S} \mathbf{H}^{H}\left(\underline{\theta}_{0}\right)\right]}}=\mathbf{0}
$$

is verified. The eigenvectors of the SDM $\mathbf{C}_{Y Y}$ are separated into signal and noise eigenvectors as in Eq. (7), and the socalled orthogonal projector onto the noise subspace is given as $\boldsymbol{\Pi}^{\perp}=\mathbf{U}_{N}^{H} \mathbf{U}_{N}^{H}$. The MUSIC processor is defined as

$$
P_{\text {MUSIC }}(\theta)=\frac{\operatorname{tr}\left[\mathbf{H}^{H}(\underline{\theta}) \mathbf{H}(\theta) \mathbf{C}_{S S}\right]}{\operatorname{tr}\left[\mathbf{H}^{H}(\underline{\theta}) \mathbf{\Pi}^{\perp} \mathbf{H}(\underline{\theta}) \mathbf{C}_{S S}\right]},
$$

such that the solution parameter occurs at the maximum of $P_{\text {MUSIC }}(\underline{\theta})$. The degree of the solution uniqueness will certainly depend on the dimension of the signal subspace since the orthogonality in Eq. (9) works as a constraint of the solutions satisfying that condition. The smaller the signal subspace dimension the larger the dimensionality of that constraint, thus, reinforcing the solution uniqueness. The dimensionality of the noise subspace will in general be high. In theory, estimates of an arbitrary accuracy can be obtained if the observation time is sufficiently long, if the signal-tonoise ratio (SNR) is adequate, and if the signal model is sufficiently accurate. The main limitations of this method are the failure to correctly estimate the parameter with a low number of observations and a poor SNR. This method has been credited as being highly sensitive to model mismatch.
Finally, it should be noted that in practice only a sample SDM $\hat{\mathbf{C}}_{Y Y}$ is available. Thus, in Eqs. (5) and (6) $\mathbf{C}_{Y Y}$ must be replaced by $\hat{\mathbf{C}}_{Y Y}$, and in Eq. (10) $\Pi^{\perp}$ must be replaced by $\hat{\mathbf{I}}^{\perp}$.

\section{E. Estimating the signal matrix}

Earlier, three matched-field processors based on the broadband data model were developed. However, the development was carried out assuming full knowledge of the signal matrix. In practice, knowledge of the emitted signal is often not available, or such knowledge may be useless due to unmodeled ocean inhomogeneities or variability in the channel response. This leads to the requirement of estimating the signal matrix $\mathbf{C}_{S S}$, which is analogous to deconvolution. ${ }^{26,27}$ Classical deconvolution assumes full knowledge of the source location and environmental parameters, which is not the case in environmental estimation problems.

The estimation of the signal matrix can be based on the signal subspace. Let $\mathbf{C}_{X X}=\mathbf{H C}_{S S} \mathbf{H}^{H}$ be the signal component of the SDM defined in Eq. (3). This can be estimated together with $\sigma_{N}^{2}$. Using the eigenvalue representation $\lambda_{1} \geqslant \ldots \geqslant \lambda_{M}$ and the orthonormal eigenvectors $\underline{u}_{i}$, $(i=1, \ldots, M)$ of $\mathbf{C}_{Y Y}\left(\underline{\theta}_{0}\right)$ spanning the signal subspace, and assuming that $\lambda_{M}>\lambda_{M+1}=\cdots=\lambda_{K L}=\sigma_{N}^{2}$, one can write

$$
\mathbf{C}_{X X}=\sum_{i=1}^{M}\left(\lambda_{i}-\sigma_{N}^{2}\right) \underline{u}_{i} \underline{u}_{i}^{H},
$$

where $M$ is the dimension of the signal subspace. Optimum estimates of the eigenvalues $\lambda_{i}$ and the eigenvectors $\underline{u}_{i}$ can be obtained from the sample SDM $\hat{\mathbf{C}}_{Y Y}\left(\underline{\theta}_{0}\right)$, and an optimum estimate of $\sigma_{N}^{2}$ can be obtained by ${ }^{28}$

$$
\hat{\sigma}_{N}^{2}=\frac{\operatorname{tr} \hat{\mathbf{C}}_{Y Y}-\sum_{i=1}^{M} \hat{\lambda}_{i}}{K L-M},
$$

which is equivalent to the arithmetic mean of the $K L-M$ smallest eigenvalues $\lambda_{i}, i=M+1, \ldots, K$ of $\hat{\mathbf{C}}_{Y Y}$. Now the estimate $\hat{\sigma}_{N}^{2}$ can be used in Eq. (11) to estimate $\mathbf{C}_{X X}$. Finally, the estimate of $\mathbf{C}_{S S}$ proceeds by filtering out the channel response:

$$
\hat{\mathbf{C}}_{S S}=\mathbf{H}^{+}\left(\underline{\hat{\theta}}_{0}\right) \hat{\mathbf{C}}_{X X}\left[\mathbf{H}^{+}\left(\underline{\hat{\theta}}_{0}\right)\right]^{H} .
$$

In Eq. (12) one problem persists: Generally $\underline{\theta}_{0}$ is unknown, and the deconvolution algorithm cannot be completed. In the framework of parameter estimation one can replace $\underline{\theta}_{0}$ with $\underline{\theta}$, making $\hat{\mathbf{C}}_{S S}$ dependent on $\underline{\theta}$, and then replacing it in the processor expressions obtained earlier. It has been observed with synthetic data that the lack of knowledge on the emitted wave form will lead to a drawback in the parameter estimation performance. Knowledge on the structure of the emitted wave form can be seen as a priori information entering the parameter estimation algorithm.

\section{SIMULATIONS}

In Sec. II, three matched-field processors based on a broadband data model were developed. The Bartlett proces- 

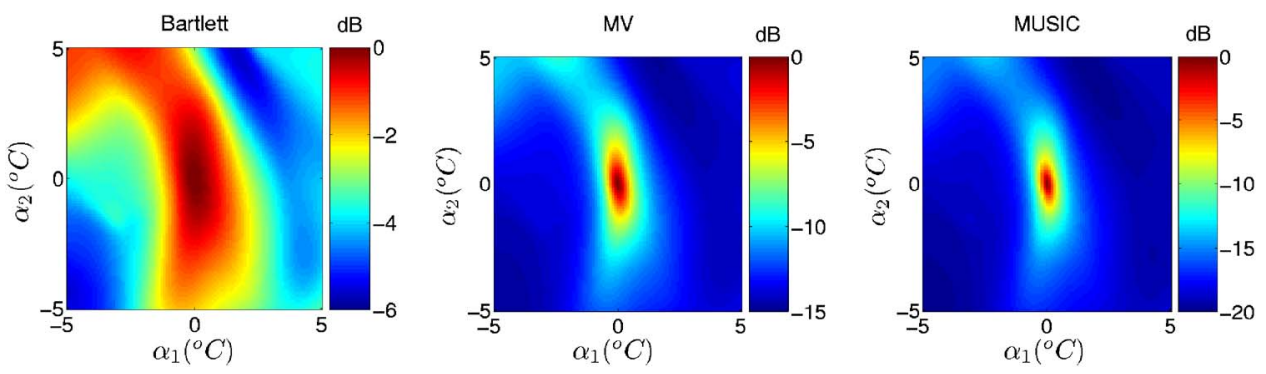

FIG. 1. (Color online) The behavior of the broadband processors for the coherent case assuming an unknown signal matrix.

sor is simply based on correlations-its implementation is straightforward. The MV and MUSIC high-resolution processors go beyond simple correlations and their computation requires additional steps. The computation of the MV processor involves the inversion of the SDM matrix, which has been noted as a difficulty in the MFP literature. The MUSIC processor involves splitting the data into signal and noise subspaces, whose correct estimation is fundamental for its success. So far, high-resolution methods have not been applied to experimental data with the purpose of performing environmental inversions. The objective of the following is to perform a simulation study in order to compare the three proposed processors covering issues such as sidelobe structure; performance for different data model assumptions; and the influence on the genetic algorithm's performance. Although all three processors will be analyzed with the same depth, the Bartlett processor will be seen as the reference in terms of performance and some more focus will be on the high-resolution methods, since these somewhat constitute a novelty for this application.

The synthetic data are generated using an environmental model for a shallow water scenario similar to that of the North Elba site. The forward problem is solved using the normal modes propagation model C-SNAP. ${ }^{29}$

\section{A. Portraying MF processors as cost functions}

A matched-field processor can be seen as a function of the hypothetical parameter vector $\underline{\theta}$, and is usually called cost function in the context of inverse problems. Concerning the behavior of a processor, assuming absence of noise and model mismatch of any type, one of the key characteristics is the ratio between the maximum value of the processor and the sidelobes, which has traditionally been considered an important issue in the context of a processor's robustness against noise. Herein the importance of that issue is reinforced in the context of the optimization problem with aid of a genetic algorithm. The main interest is to illustrate how the matched-field processors obtained in Sec. II compare in terms of sidelobe attenuation and resolution, and how the assumptions of known or unknown wave form, or the assumption of coherent or incoherent signals impact on these characteristics. This can be carried out numerically by calculating each cost function as a function of physical parameters of interest for a given scenario example. The source was supposed to be at a $6 \mathrm{~km}$ range and at a $60 \mathrm{~m}$ depth, and receivers were at depths 15,60 , and $75 \mathrm{~m}(L=3)$. The acoustic field was considered for frequencies 400, 450, and $500 \mathrm{~Hz}(K=3)$. The spectral density matrix was computed

using Eq. (3). The noise power $\sigma_{N}^{2}$ was set as the mean of the first $K$ eigenvalues $\lambda_{k}$ of the $\mathbf{C}_{X X}$ matrix (the SDM of the signal component) in order to obtain autofrequency SDMs with the same SNR, in both coherent and incoherent cases. The noise is assumed uncorrelated both across space and frequency. Note that under the assumption of coherent signals the eigenvalues of $\mathbf{C}_{X X} \lambda_{1}>0$ and $\lambda_{2}=\cdots=\lambda_{K}=0$, and for the incoherent case, in general, $\lambda_{k}>0$. Note also that for computing the SDM, $\mathbf{C}_{S S}=\mathbf{1}$ for the coherent case, and $\mathbf{C}_{S S}$ $=\mathbf{I}$ for the incoherent case. Some more remarks are necessary before proceeding: (a) In Sec. II, the emitted wave form will always be represented by second-order statistics, i.e., by matrix $\mathbf{C}_{S S}$; (b) in Sec. II A, for computing the cost functions, and, in particular, for estimating the signal matrix or for estimating the noise subspace, it is assumed that the dimension of the signal subspace is known, which is 1 in the coherent case and $K$ in the incoherent case.

The cost functions were computed as a function of two coefficients $\alpha_{1}$ and $\alpha_{2}$ used to parametrize the temperature profile (see the following details) with $\left[\alpha_{1} \alpha_{2}\right]^{T}=[00]^{T}$ as true parameter values. Figure 1 shows the matched-field response of the three BB processors computed for the case assuming coherent spectral components and unknown signal structure. The three plots respectively correspond to implementations of Eqs. (5), (6), and (10), together with Eq. (12) for estimating the signal matrix. Observing Fig. 1, the plot on the left contains a very smooth function as is typical for the Bartlett processor, with a variation between minimum and maximum values of $6 \mathrm{~dB}$. The plot in the middle corresponds to the coherent MV processor, which is clearly superior to the Bartlett processor in terms of sidelobe attenuation, with values ranging by about $15 \mathrm{~dB}$. Finally, a plot corresponding to the MUSIC processor was computed. This processor has the best sidelobe attenuation performance of all, with values ranging between -20 and $0 \mathrm{~dB}$. The reader might ask how this was done if this processor approaches $\infty$ as the parameter vector $\underline{\theta}$ approaches the true value, under the conditions used for generating the synthetic data. It is possible to portray the MUSIC processor such that its maximum is 1 by

$$
P_{\text {MUSIC }, 1}=\frac{\gamma}{\gamma+\frac{1}{P_{\text {MUSIC }}} .}
$$

It can be seen that $P_{\text {MUSIC, } 1} \rightarrow 1$ as $P_{\text {MUSIC }} \rightarrow \infty$. This modification has two effects: (1) The maximum value that can be attained is a known finite value; and (2) implementation allows for smoothing the processor. Small values for $\gamma$ will produce a peaky function, while large values for $\gamma$ will pro- 
TABLE I. Peak-to-surface average ratio obtained for the different processors.

\begin{tabular}{lcccc}
\hline \hline & $\begin{array}{c}\text { Coherent } \\
\text { known } \\
\text { signal }\end{array}$ & $\begin{array}{c}\text { Coherent } \\
\text { unknown } \\
\text { ignal }\end{array}$ & $\begin{array}{c}\text { Incoherent } \\
\text { known } \\
\text { signal }\end{array}$ & $\begin{array}{c}\text { Incoherent } \\
\text { unknown } \\
\text { signal }\end{array}$ \\
\hline Bartlett & 2.23 & 1.86 & 1.64 & 1.58 \\
MV & 17.9 & 14.5 & 6.08 & 5.41 \\
MUSIC & 53.0 & 41.9 & 41.9 & 37.2 \\
\hline \hline
\end{tabular}

duce a smooth function. In this study, $\gamma$ has been always set to 0.01 .

Table I summarizes the results obtained in terms of peak-to-surface average ratio for different combinations of coherent/incoherent and known/unknown signal matrix. This measure is the ratio between the surface maximum and its average $\mathrm{MF}$ response. It is easy to conclude that there is an increasing discriminating potential when presenting the developed methods in that sequence. The coherent MV processor shows the highest effectiveness in comparison with its incoherent counterpart. For the other two processors coherent processing with unknown signal gives similar performance to incoherent processing with known signal.

\section{B. Error performance}

The next issue to be investigated is how the coherent processors perform against the number of signal snapshots $N$ for a given SNR. Here the parameter to be estimated is $\alpha_{1}$, whose true value is 0 . Once again the three frequencies/three receivers case is taken with a $\mathrm{SNR}=0 \mathrm{~dB}$. Figure 2 shows plots with computations of the RMSE as a function of the number of snapshots $N$ based on 100 estimates of the parameter. In no case is the Cramer-Rao lower bound attained. Figure 2(a) shows the RMSE with known signal matrix and signal subspace dimension. The MUSIC and Bartlett processors perform similarly for a known signal matrix. The MV processor has poor performance for low number of snapshots and recovers comparatively to the others as the number of snapshots increases. Finally, in the case of unknown signal matrix and signal subspace dimension, Fig. 2(b) shows how the low number of snapshots can impact on the performance of subspace-based methods, in particular, on the separation of the subspaces, in a conjunction with low SNR and low number of snapshots. For the other two processors, working with unknown signal slightly increases the RMSE.

The difficulties seen with the high-resolution methods are related to poor estimates of the eigenvalues $\lambda_{i}$. The data model assumes that the eigenvalues associated with the noise subspace are all equal. However, if $N$ is finite, those will be different with probability 1 . The MV processor requires inversion of the SDM, whose accuracy depends on the eigenvalues' estimates. Since it weights the eigenvector associated with the smallest eigenvalue most heavily, and this is the least stable vector because it has the least energy and must be orthogonal to all others, a small $N$ is certainly a source of relatively poor performance. Concerning the MUSIC processor, the problem arises when the signal space is to be split into the signal and noise subspaces. This important issue is
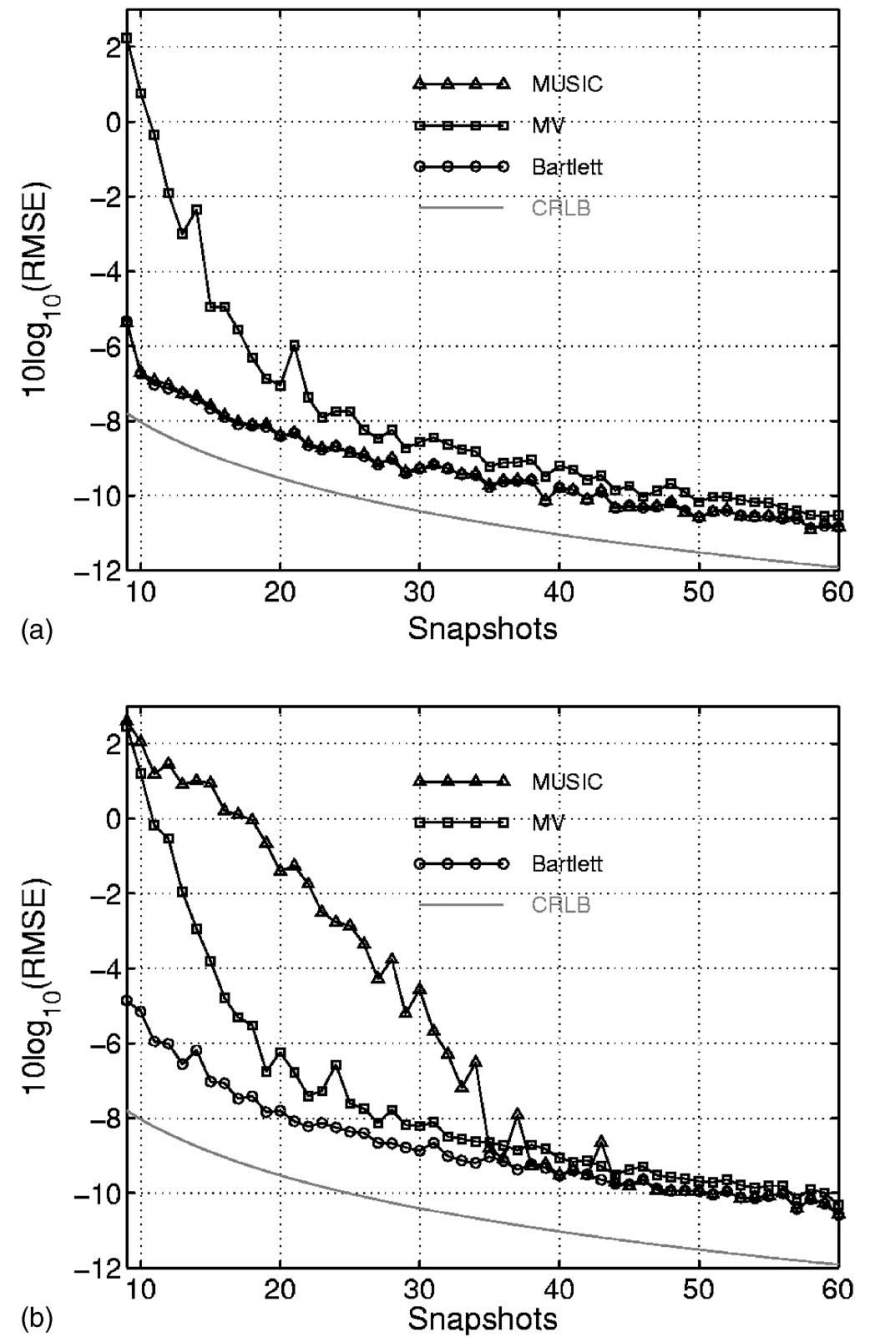

FIG. 2. RMSE as a function of the number of snapshots for the three processors and the coherent model Cramer-Rao lower bound under comparison: (a) With known signal matrix and (b) with unknown signal matrix and signal subspace dimension.

illustrated in Fig. 3, which shows in (a) the computed eigenvalues for two particular cases, one using 9 signal realizations, and the other using 11. The signal subspace dimension is estimated using the MDL criterion. ${ }^{30-32}$ The former case yields a signal subspace with dimension eight, although a deterministic signal component is used for data generation. Note that the smallest eigenvalue has a very high ratio to its predecessor. The latter case, $N=11$, yielded a signal subspace with dimension one-in that case the ratios between contiguous higher-order eigenvalues are reduced. Figure 3(b) shows the average eigenspectrum when the number of realizations varies from 9 to 20. For each case 100 realizations of the eigenspectrum were computed and averaged. On average, the eigenspectrum tends to become flattened as $N \rightarrow \infty$. Finally, Fig. 3(c) shows the average order estimate obtained using the same data as in Fig. 3(b), applying the MDL information criterion. For the minimal number of signal realizations (9) the average order obtained is about 3; for 10 realizations it is about 2; for 11 realizations or more it estimates on average the correct value, which is 1 . This exercise illustrates the potential impact of the number of signal realizations on methods relying on the eigendecomposition of the data. 

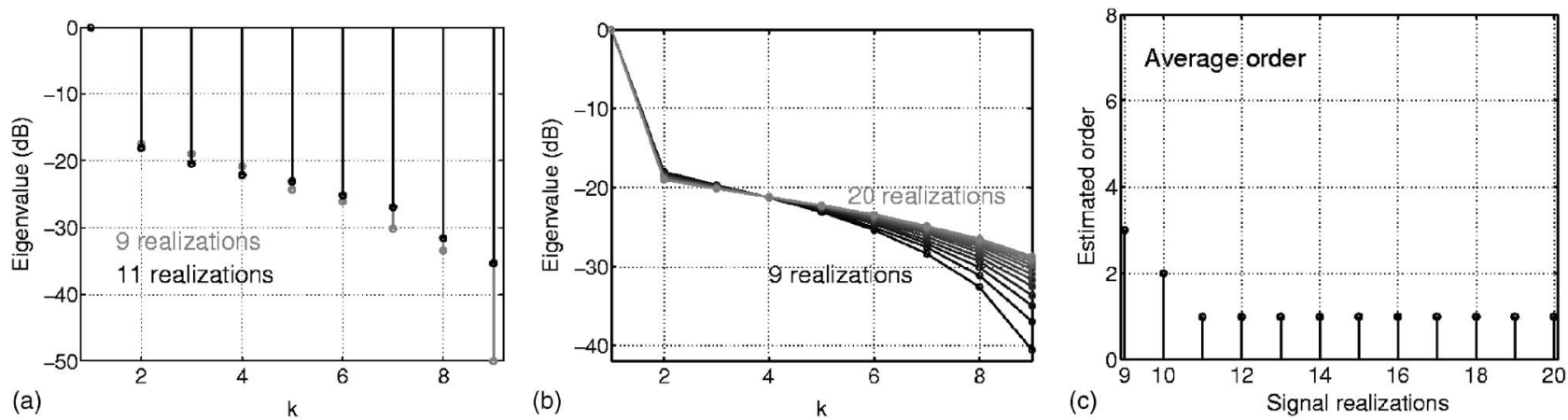

FIG. 3. Eigenspectra for finite number of signal observations: (a) Comparison of two eigen-spectra using $N=9$ (gray) and $N=11$ black; (b) average eigenspectrum for a varying number of signal realizations; and (c) average order estimation for a varying number of signal realizations.

\section{Global search}

In Sec. III A it is shown that all three proposed MF processors significantly differ in their ability of attenuating sidelobes. The next case study is to conclude about the comparative performance of the processors when the environmental estimation problem includes multiple unknown parameters. This problem is usually solved with the aid of a global search method such as a genetic algorithm. The idea is to find out whether high-resolution methods can improve the convergence of meta-heuristic search methods due to their reduced sidelobe structure. Sidelobes competing with the main peak may be seen as false attractors that cause difficulties to any meta-heuristic search method in converging to the solution maximizing the cost function.

The data are generated and inverted 50 times using a genetic algorithm. The inversion search space regarded the water column and the seafloor properties, but array tilt was also included. The signal matrix and the signal subspaces dimension were known. The general conditions for synthetic data generation are the same as those used earlier with SNR of $0 \mathrm{~dB}$ and 16 snapshots.

A posteriori distributions can provide insight into the performance of the environmental inversion process. These distributions emphasize the variability of each parameter over the search interval, which is intimately related to the ambiguity pattern of the cost function used and the sensitivity to each parameter. Figure 4 shows the a posteriori distributions obtained for the three methods. To obtain these distributions the individuals of the last generation of all independent populations are merged and histograms are computed from the parameter vectors represented by those individuals.

The idea of showing all these distributions is to obtain a global comparison of the three processors in terms of convergence rather than performing a detailed analysis. The MUSIC processor clearly has the narrowest distributions. In fact, that processor contributed to improving the convergence of the genetic algorithm, supporting the belief that a massive sidelobe structure causes difficulties in terms of the population convergence. The MV processor appears to be more uncertain, which is rather attributed to the problem of inverting the SDM with a small $N$ than to the sidelobe structure. Finally, the distributions obtained with the Bartlett processor are significantly more spread out over the search interval than the others, which is attributed to its massive sidelobe structure causing difficulties for the search algorithm in converging to the true solution. From the a posteriori distributions, model estimates based on the distribution peak, called Maximum A Posteriori (MAP) estimates, can be obtained. The MUSIC processor produced more reliable MAP estimates, since its parameter distributions are the most compact, and all parameters, except sediment upper speed and subbottom density, have a posteriori distributions with a peak close to the true parameter value (indicated by the gray asterisk).

\section{ENVIRONMENTAL INVERSION OF EXPERIMENTAL DATA}

\section{A. The MREA'03 sea trial}

The Maritime Rapid Environmental Assessment 2003 (MREA'03) sea trial took place from 26 May to 27 June 2003, in the Ligurian Sea, with target areas North and South of Elba Island. This paper considers only the acoustic experiment held on 21 June $2003^{33}$ whose area of operation was North of the Elba Island as shown in Fig. 5.

\section{The deployment geometry}

On 21 June, the AOB was deployed on a free drift configuration with very favorable weather conditions in an area of mild bottom range-dependency, attaining a variability of 20 m over some acoustic tracks. The experimental setup consisted of a towed acoustic source and a free drifting vertical line array with receivers at nominal depths of $15,60,75$, and $90 \mathrm{~m}$. Figure 5(b) shows the bathymetry in the interior of the white box depicted in the map of Fig. 5(a) together with the source ship navigation and AOB drift estimated from GPS recordings. The acoustic buoy was deployed at 09:01 GMT and recovered at about 15:16 GMT. During this time the buoy drifted about $1.7 \mathrm{~km}$ away to the Southeast of the point of deployment, at approximate average displacement of $4.5 \mathrm{~m} / \mathrm{min}$ (white dashed line).

The acoustic source was deployed immediately after the acoustic buoy and towed by the RV Alliance to West where it was stalled between times 09:53 and 11:05 GMT. Then RV Alliance steadily moved to the East and performed the geometry shown in Fig. 5(b) (white solid line) until source recovery. Figure 6(a) shows the GPS estimated range be- 

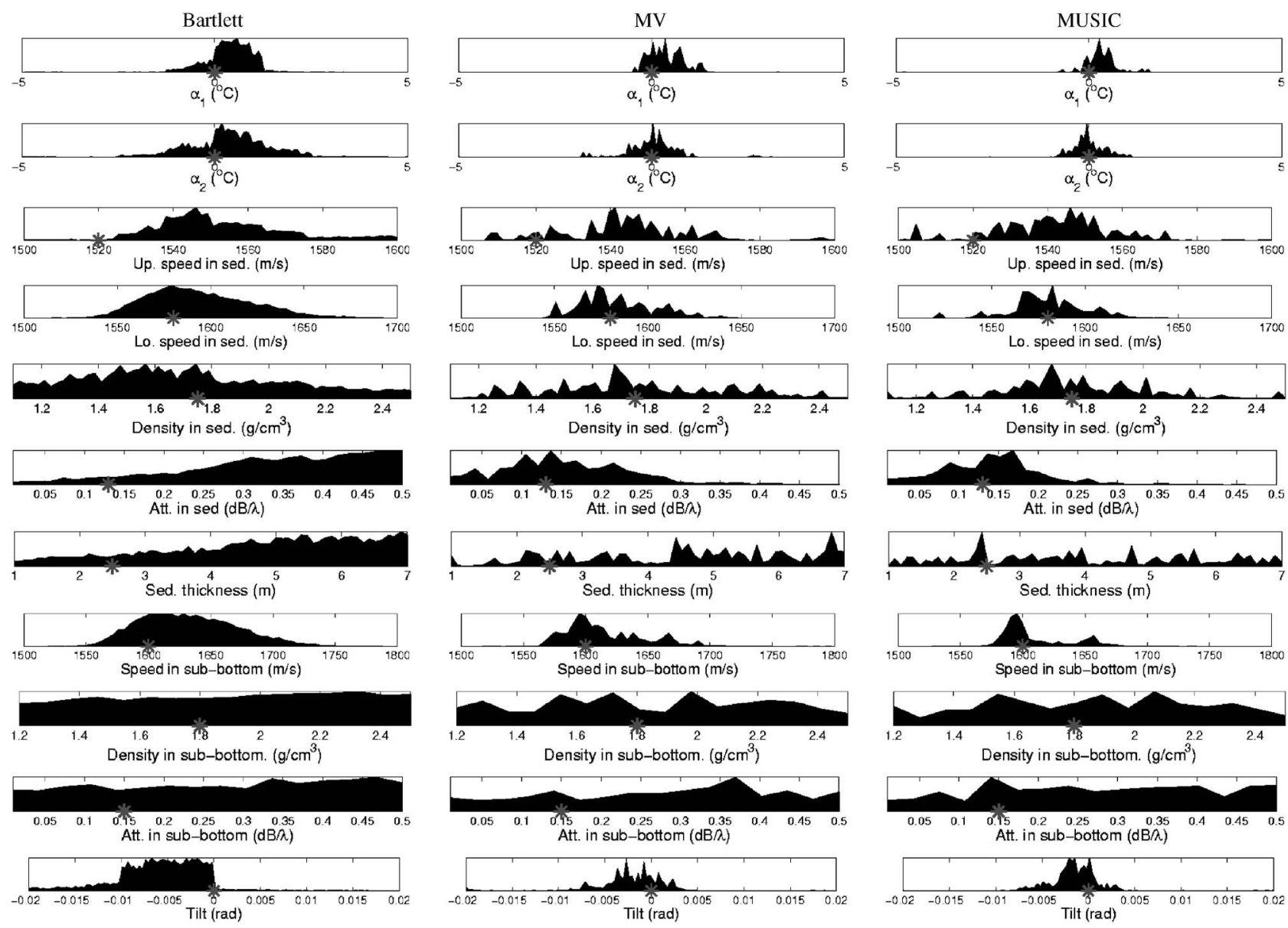

FIG. 4. A posteriori probability distributions for each parameter based on the last generation of 50 independent populations. Each column corresponds to processors entering the comparison. The gray asterisks indicate the correct parameter value.

tween source and receiver and Fig. 6(b) the source depth. The acoustic source was deployed at a variable depth, between 54 and $106 \mathrm{~m}$, depending on ship speed.

\section{Acoustic signals}

The emitted wave forms consisted of 2-s LFM chirps emitted in two frequency bands. The $A 1$ and Aldouble chirps are in the band $500-800 \mathrm{~Hz}$. The signals differ in terms of repetition interval and duty cycle: $A 1$ lasts for $2 \mathrm{~s}$ and has a repetition interval of $8 \mathrm{~s}$ hence a duty cycle of $25 \%$; Aldouble lasts for $4 \mathrm{~s}$ and has a repetition interval of $10 \mathrm{~s}$ hence a duty cycle of $40 \%$. The objective of emitting the Aldouble wave form was to increase the number of signal realizations in a given observation interval, which may significantly impact on the performance of some matched-field processors. The $A 2$ chirp is in the band $900-1200 \mathrm{~Hz}$. Table II shows the emission schedule indicating the periods during which each wave form was emitted. The signals were received at a vertical array containing four hydrophones at nominal depths of 15, 60, 75, and $90 \mathrm{~m}$. Figure 7 shows an example of receptions of the $A 2$ chirps at time 11:45 GMT collected at the third receiver. The data are extremely clean without blanks and interruptions, which results from the AOB's local storage capability. However, it was found that the acoustic data collected by the deepest hydrophone was very noisy most of the time, possibly due to deployment issues that were not well understood. For this reason it was decided to not consider that hydrophone in the present work. A final remark is that a channel fading effect that significantly reduces the signal received on the top most hydrophone was noticed, probably due to the effect of the thermocline.

\section{Environmental data measurements}

Regarding the inversion of the acoustic data collected on 21 June, 95 conductivity-temperature-depth (CTD) measurements taken during the days 16,17 , and 19 June taken at the positions marked by the black circles in Fig. 5 are considered in this study. No CTD measurements were performed during that acoustic experiment since RV Alliance was towing the source. There is a significant difference in scale between the acoustic (white box) and the oceanographic survey, as the latter was set up for different purposes. Those CTD measurements are being taken as an attempt to cope with the difficulty in sampling the ocean volume, both in time and space, and obtain representative a priori oceanographic data as an input to the acoustic inversion problem. It is of concern to what extent these historical data collected several days before may be representing the oceanography of the target day. Figure 8 shows the measured temperature profiles with two empirical orthogonal functions (EOF) representing more than $80 \%$ of the water column variability. The water column 

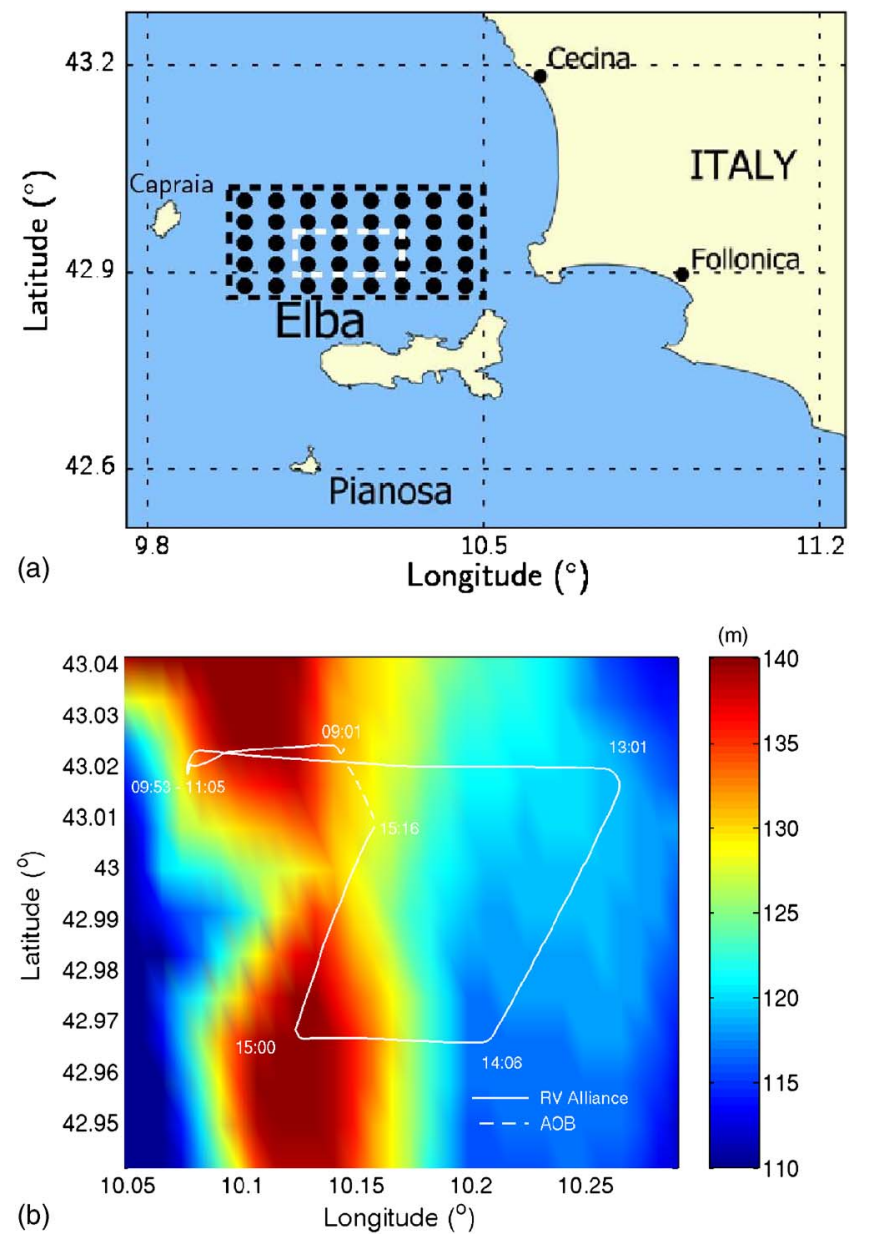

FIG. 5. (Color online) The Maritime Rapid Environmental Assessment 2003 (MREA'03) experimental area: (a) Black circles indicate the sampling grid setup for the CTD measurements used in this study, and the dashed white box limits the area where the acoustic experiment of 21 June took place and (b) GPS estimated source ship navigation (white solid curve) and AOB drift (white dashed curve) during the deployment of 21 June.
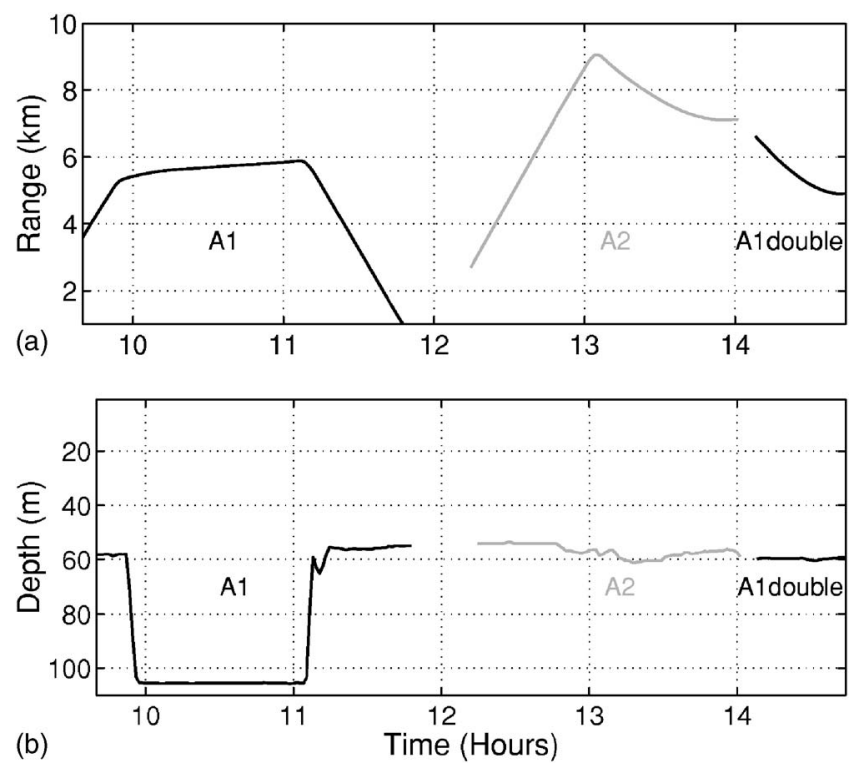

FIG. 6. Source range (a) and depth (b) measured during the deployment of 21 June. The curves are broken indicating change of the emitted wave form. A1, A2, and A1double denote the wave forms emitted in each interval.
TABLE II. Signal emission schedule on 21 June. The times are in GMT.

\begin{tabular}{lccc}
\hline \hline & Al & A2 & A1double \\
\hline Start & $09: 40$ & $12: 14$ & $14: 07$ \\
End & $11: 47$ & $14: 01$ & $14: 44$ \\
\hline \hline
\end{tabular}

temperature is then modeled as a sum of the mean temperature (thick curve) and the two EOFs weighted by associated EOF coefficients $\alpha_{n}$. It is useful to measure the coefficients for the historical data available in order to obtain hints on their range of variation. In the present case $\alpha_{1}$ varied in the interval from -15 to 15 , and $\alpha_{2}$ varied in the interval from -5 to 5 temperature profiles considered.

\section{The environmental model}

One of the tasks with the largest impact on the final result is the choice of an adequate environmental model to represent the propagation conditions of the experiment. This choice is generally the result of a compromise between a detailed, accurate, and parameter full model and a light model ensuring a rapid convergence during the processing. The baseline computer model adopted for the MREA'03 was built based on the segmentation of archival bathymetric information along the source-receiver cross sections at different times. As shown in Fig. 5 the bathymetry in the experimental area is accurately known. The water depth at the AOB deployment site was approximately $120 \mathrm{~m}$ and the maximum depth at the emitting source was $140 \mathrm{~m}$. The baseline geoacoustic properties were drawn from previous studies in that area. ${ }^{10,34}$ The baseline model consists of an ocean layer overlying a sediment layer and a bottom half space with the bathymetry assumed range dependent, as shown in Fig. 9. The sound-speed profile was calculated using the Mackenzie formula with the mean temperature and mean salinity profiles as inputs (see Fig. 8).

For the purposes of the inversion the forward model was divided into four parameter subsets-water column temperature, sediment, subbottom, and geometric parameters. The temperature in the water column is parametrized by the two EF coefficients as discussed earlier.

\section{B. Results}

The following reports on environmental inversions of the experimental acoustic data for water column and seafloor properties using the broadband processors proposed in Sec. II, and the comparison of their estimation performance.

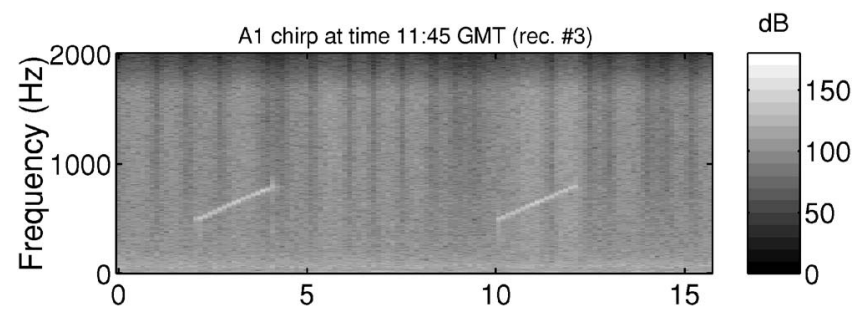

FIG. 7. Example of an A1 chirp received on the AOB. 

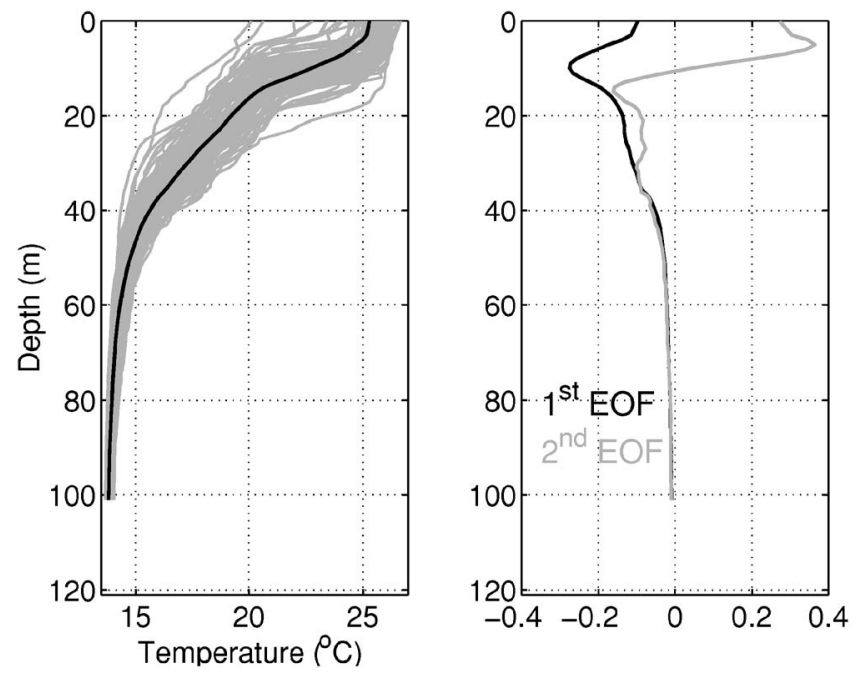

FIG. 8. CTD-based data used for temperature estimation taken during 16, 17, and 19 June Temperature profiles with mean profile in solid black (left) and representative empirical orthogonal functions (EOF) computed from the temperature profiles (right).

\section{Frequency clustering}

The proposed BB matched-field processors are based on the $K L \times K L \mathrm{SDM} \mathbf{C}_{Y Y}$ measuring cross correlations of the acoustic field across space and frequency. Both the MV and MUSIC processors require $\mathbf{C}_{Y Y}$ to be full-rank, i.e., $N \geqslant K L$. If an observation window of $80 \mathrm{~s}$ is taken, then the MREA'03 data set provides $N=10$ for the $A 1$ and $A 2$ intervals, and $N=16$ for the Aldouble interval. Given a number of receivers $L=3$ and $N=10$, one can choose a number of frequencies $K=3$ in order to assure that full-rank SDMs are obtained in all emission intervals. In order to use an increased number of frequencies while assuring that the SDM is full rank one can use an alternative matched-field processor output given as

$$
P^{\mathcal{N}_{g}}(\underline{\theta})=\frac{1}{\mathcal{N}_{g}} \sum_{n=1}^{\mathcal{N}_{g}} P\left(\underline{\theta}, \underline{\omega}_{n}\right) .
$$

This is an incoherent average over $\mathcal{N}_{g}$ coherent frequency clusters $\underline{\omega}_{n}$, where $P\left(\underline{\theta}, \underline{\omega}_{n}\right)$ is a given matched-field processor. The question is how to choose the frequency clus-

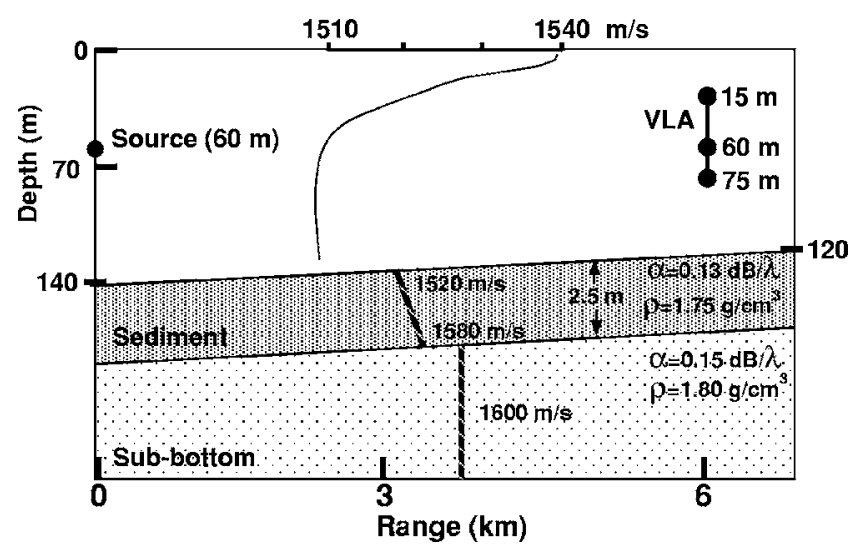

FIG. 9. Baseline model for the MREA'03 sea trial. All parameters except water depth are range independent.
TABLE III. GA settings for environmental inversion.

\begin{tabular}{lc}
\hline \hline Parameter & Setting \\
\hline Generations & 30 \\
Population size & 200 \\
Independent populations & 1 \\
Mutation probability & 0.004 \\
Crossover probability & 0.9 \\
Number of crossover points & 4 \\
\hline \hline
\end{tabular}

ters. As the idea is to use coherent processors one can carry out an optimization aimed at finding $\operatorname{SDMs} \mathbf{C}_{Y Y}\left(\underline{\omega}_{n}\right)$ with the most coherent frequencies. Ideally, one would cluster perfectly coherent frequencies, which results in a signal subspace with dimension equal 1 . Thus, it appears natural to implement an optimization scheme based on the minimization of the signal subspace dimension, using an information criterion as cost function. Since these criteria are not reliable for $N \approx K L$ it was decided to choose $\mathcal{N}_{g}$ cluster with the largest $\lambda_{1} / \lambda_{2}$ - the ratio between the two largest eigenvalues of $\mathbf{C}_{Y Y}\left(\underline{\omega}_{n}\right)$ is a simplified measure of the signal's coherence.

This is a preprocessing step that performs a selection of frequency combinations based on a coherence criterion. In this study a number of clusters $\mathcal{N}_{g}=7$ will be used, and the optimization was carried out using a frequency resolution of $4 \mathrm{~Hz}$. In order to assure spectral diversity, frequencies in a cluster are separated by at least $52 \mathrm{~Hz}$.

\section{Data processing procedure}

Several steps are performed until the inversion is complete:

(1) Frequency selection based on the $\lambda_{1} / \lambda_{2}$ optimization criterion.

(2) Acoustic field inversion for water column and seafloor properties, and geometric nuisance parameters.

(3) Inversion validation by means of source localization with large search bounds using the estimated environmental models.

(4) Reconstruction of physical parameters of interest using only environmental estimates validated in step (3).

In step (2) the unknown parameters are divided into water column ( $\alpha_{1}$ and $\alpha_{2}$ EOF coefficients), sediment (upper and lower compressional speeds, density, attenuation, and thickness), and subbottom (compressional speed, density, and attenuation). Additionally, geometric parameters, array tilt, and receiver depth are included. These parameters are regarded as nuisance parameters, since there is no interest in their estimates once the inversion is finished. The inversion is posed as an optimization problem solved with aid of a genetic algorithm (GA). ${ }^{35}$ The parameter vector is coded into a 68-bit chain, which results in a search space size approximately equal to $2.95 \times 10^{20}$. The GA settings are summarized in Table III. Since there is a new time bin every 80 s only a single population is used for each inversion, which is sufficient to achieve the main objective of comparing the proposed MF processors' inversion performance. 
TABLE IV. Rates of successful localization (\%) for the different processors and different wave forms.

\begin{tabular}{lcccc}
\hline \hline Processor & A1 & A2 & A1double & Overall \\
\hline Bartlett & 22.7 & 38.3 & 51.9 & 32.7 \\
MV & 22.7 & 29.6 & 85.2 & 35.1 \\
MUSIC & 39.0 & 53.1 & 70.4 & 48.3 \\
\hline \hline
\end{tabular}

Step (3) is to validate the model estimates obtained in step (2) by means of range-depth source localization. This step is based on the accurate knowledge on source range and depth available and on the fact that source position is on top of the parameter hierarchy. It is assumed that if the environmental estimates are not accurate then the source cannot be properly located. Performing source localization with large search bounds should give an indication on the quality of a given environmental estimate.

Finally, step (4) is to produce the final environmental estimates using only those estimates validated in step (3).

\section{Environmental inversion: Comparison of three MF processors}

Here, the three BB processors will be applied to experimental field data. The whole data set collected on 21 June will be inverted with each processor. In Sec. III C, inversions on synthetic data with a GA indicated that high-resolution processors may contribute to improve the convergence to the true solution. The comparison performed here may also serve the purpose of understanding how these processors behave in a real situation with the inherent model mismatch. The maximum source-receiver range is $9 \mathrm{~km}$, which is more than 70 water depths, possibly giving rise to environmental mismatch.

The inversion is carried out assuming an unknown signal matrix $\mathbf{C}_{S S}$. Since the frequencies are optimized in the sense of clustering those with highest coherence, it is assumed that the signal subspace dimension is always one, although source and receiver are moving most of the time. The processing is, therefore, considered broadband coherent with unknown wave form.

No ground truth measurements are available for evaluating the inversion performance achieved. Thus, one can refer directly to step (3), the validation step, and analyze those results. Source localization along time was performed within search bounds from 1 to $10 \mathrm{~km}$ in range, and from 1 to $110 \mathrm{~m}$ in depth. The source is admitted as correctly localized if the error both in range and depth is simultaneously less than $5 \%$ of the search interval amplitude. In other words the maximum must fall in a $0.9-\mathrm{km}$-long and $11-\mathrm{m}$-wide rectangle centered on the true location. Table IV shows the rate of successful localization achieved with each processor. The localization rate was computed for each emission interval due to the different signal characteristics, and then summarized on the rightmost column as an overall result. Considering the overall results, the MUSIC processor clearly outperforms the other two processors, as the localization rate increases with the processors' resolution. A more detailed analysis of the result allows for making the following re-
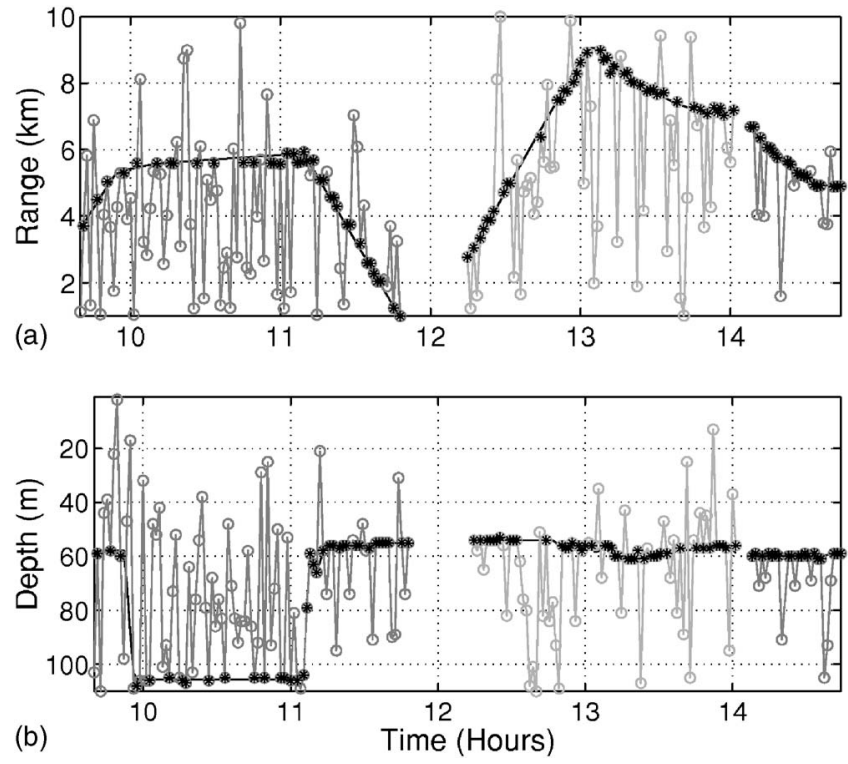

FIG. 10. Source localization obtained with the MUSIC processor. Source range (a) and source depth (b). True location is given by the black curve in the background. The gray curves with circles are the source localization results. The black asterisks indicate the successful localizations.

marks: (i) The Al interval was the most difficult. Close inspection shows that the localization rate is clearly lower with the source stalled at $105 \mathrm{~m}$ (during time 10:00 to 11:00) in the three cases. (ii) The MV processor performed remarkably well during the Aldouble interval. It appears that this processor performed particularly well with the number of signal realizations $N=16$ provided by that data portion, while showing difficulties in the other data portions providing $N=10$ (which is consistent with the synthetic study). (iii) The source was located both in range and depth at ranges up to $9 \mathrm{~km}$, as indicated by the black asterisks in Fig. 10 .

Figure 11 shows the environmental inversion results obtained with the MUSIC processor. The reason for showing the run performed with the MUSIC processor was its overall superiority in terms of source localization, and the belief that this means that the environmental estimations are also of superior quality in comparison with those obtained with the other processors. The circles filled with an asterisk correspond to inversions with successful localization.

It can be seen in plot (a) that from time 10:00 to time 11:00 the estimate of the $\alpha_{1}$ EOF coefficient, a leading parameter, varies over the entire search interval. When the source moves and goes up to approximately midwater column, the variability substantially reduces, with the estimates becoming confined in the interval -10 to 10 . During $A 2$ the estimates continue in the interval -10 to 10 at the beginning but then during the remaining part the estimates are in the upper half of the search interval between 0 and 15. Finally, the $\alpha_{1}$ estimates in the Aldouble period are clearly in the interval -10 to 10 . The second EOF coefficient was searched in an interval with amplitude less than half of that for the first one. The EOFs used to model the water column differ significantly only in the first third of the water column, while they coincide for the remaining depth. On the other hand only one receiver is in place in the first third of the water 

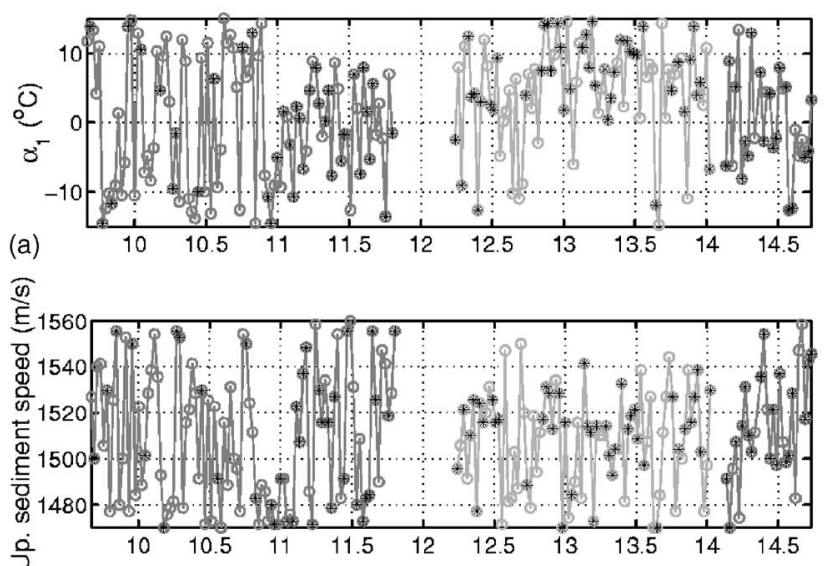

(c)

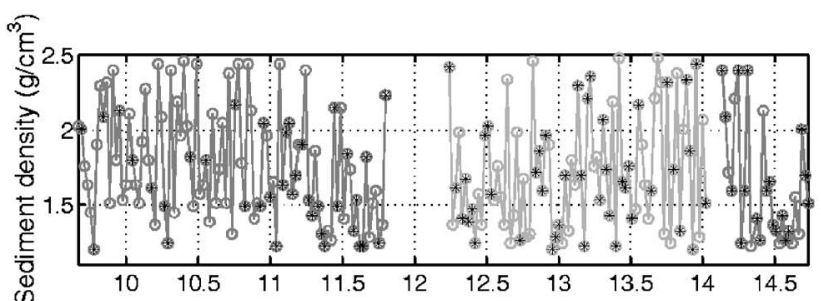

$(e)$

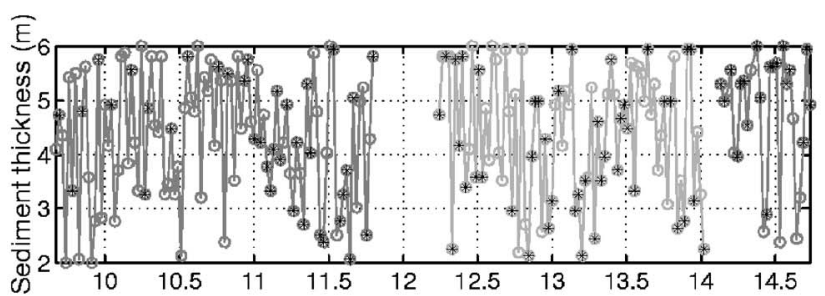

(g)

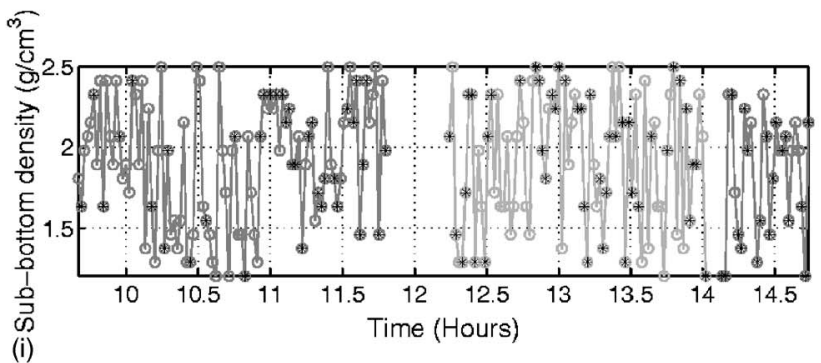

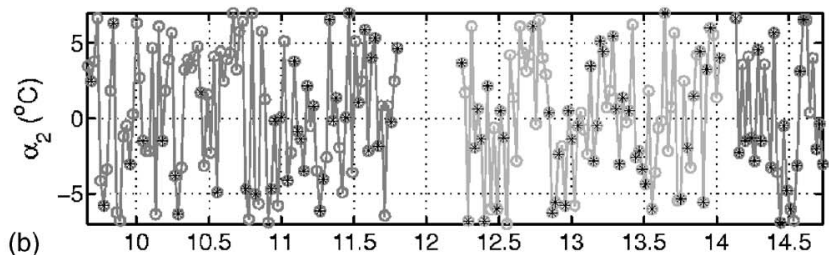

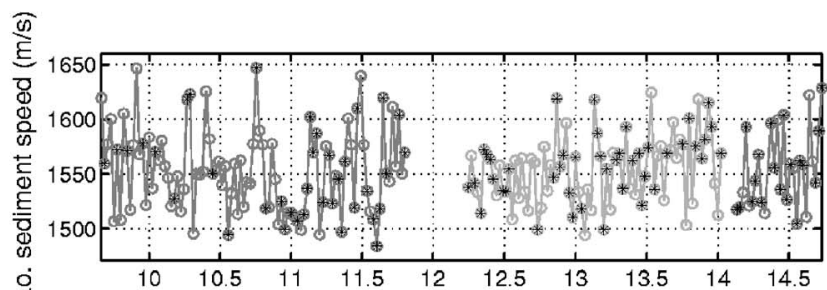

(d)

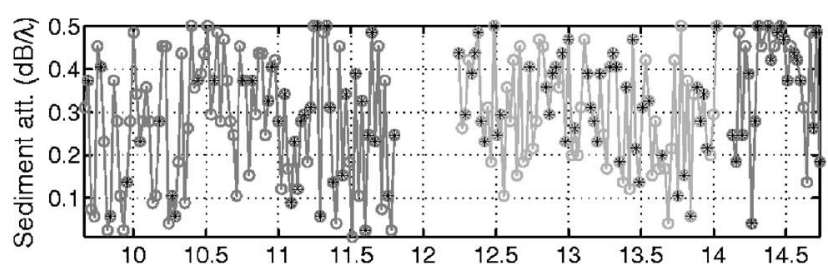

(f)

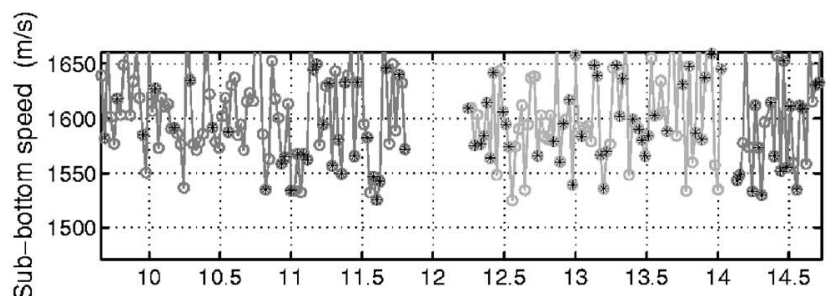

(h)

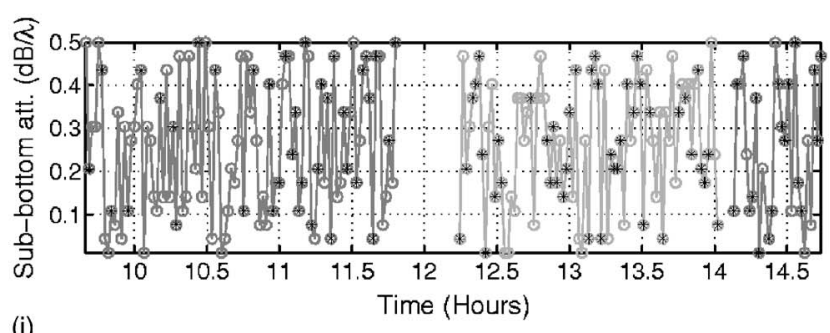

FIG. 11. Model parameter estimates obtained via acoustic data inversion using the BB MUSIC processor. Water column [(a) and (b)]; sediment [(c)-(g)]; and subbottom $[(\mathrm{h})-(\mathrm{j})]$. The black asterisks indicate model estimates allowing for successful source localization in the validation step.

column. In Ref. 36 it is shown that these circumstances lead to an ambiguous cost function regarding the $\alpha_{i}$ coefficients, resulting in a poor solution uniqueness.

Concerning the seafloor parameter estimates, most of them appear to be unstable, making it nearly impossible to draw some value from the plots of Fig. 11. Nevertheless, the compressional speeds in sediment and subbottom appear to be fairly restricted to subintervals in the search interval with aid of the source localization step. In order to obtain a single estimate for each of the seafloor parameters, a posteriori distributions based on the individuals of the last generation of each inversion allowing for correct source localization (43 out of 81 inversions) were computed considering only the $A 2$ emission period (see Fig. 12). Sediment and subbottom compressional speeds, and sediment attenuation are relatively compact, each with a peak close to the baseline value. Also, sediment density and thickness show an outstanding distribution peak. The sediment attenuation distribution, although compact, is concentrated at the upper search bound far from the baseline value. Table V contains the MAP estimates for the seafloor parameters, together with the baseline values and a measure of the estimation reliability, which is the standard deviation of the a posteriori distribution divided by the search interval length. The MAP estimates of the compressional speeds are in fair agreement with the baseline values. Sediment density and thickness are also credible.

Finally, it can be remarked that during the $A 2$ emission period, the MUSIC processor produced the most reliable environmental estimates. During the Aldouble emission period the most reliable environmental estimates were produced by 
TABLE V. Baseline seafloor parameters, parameter MAP estimates on 43 GA populations, and a reliability measure.

\begin{tabular}{lccc}
\hline \hline Mode parameter & Baseline & MAP & Reliability \\
\hline Sediment & & & \\
Up comp speed $(\mathrm{m} / \mathrm{s})$ & 1520 & 1527 & 0.22 \\
Lo comp speed $(\mathrm{m} / \mathrm{s})$ & 1580 & 1570 & 0.17 \\
Density $\left(\mathrm{g} / \mathrm{cm}^{3}\right)$ & 1.75 & 1.48 & 0.27 \\
Attenuation $(\mathrm{dB} / \lambda)$ & 0.13 & 0.44 & 0.20 \\
Thickness $(\mathrm{m})$ & 2.5 & 4.92 & 0.27 \\
Subbottom & & & \\
Comp speed $(\mathrm{m} / \mathrm{s})$ & 1600 & 1618 & 0.14 \\
Density $\left(\mathrm{g} / \mathrm{m}^{3}\right)$ & 1.80 & 1.29 & 0.31 \\
Attenuation $(\mathrm{dB} / \lambda)$ & 0.15 & 0.40 & 0.29 \\
\hline \hline
\end{tabular}

the MV processor. This supports the close relation between the rate of source localization and the quality of the environmental estimation, and the choice of source localization as a validation tool.

\section{CONCLUSIONS}

Acoustic data were collected using a prototype of a light acoustic receiving system, an AOB, developed to meet operational requirements such that it consisted of a vertical line array with only three operating receivers. One of the objectives of these acoustic data was to evaluate the performance of newly adapted field inversion methods for the estimation of water column and geoacoustic properties using such a light acoustic receiving system.

The application of genetic algorithms for the estimation of model parameters has been shown to be very effective on several occasions. However, when a sparse receiving array is used the inversion problem becomes heavily ill-conditioned. Traditionally, the difficulty of solving an ill-conditioned problem has been associated with model mismatch and noise, which may lead to the situation where the optimum is not in coincidence with (or even close to) the true model parameters.

This paper associates this difficulty with the application of a genetic algorithm to carry out the optimization, specifically due to the existence of many local extrema in concurrence with the main peak, together with the typically large search space of a multiple environmental estimation problem. In order to cope with this difficulty broadband matchedfield processors, specifically, high-resolution processors, are proposed for their increased ability in attenuating sidelobes.

A Bartlett, a minimum-variance, and MUSIC processor, all truly broadband rather than a superposition of multiple frequencies since they are based on a broadband data model, were first compared in terms of estimation performance with synthetic data. It was concluded that in the case of an exhaustive search of the unknown parameter, the highresolution processors were unable to outperform the Bartlett processor. The main difficulty of the application of the highresolution processors is related to a reduced number of signal realizations to compute the sample spectral density matrix (SDM). In the case of a multiparameter inversion problem the comparison shows that the high-resolution methods
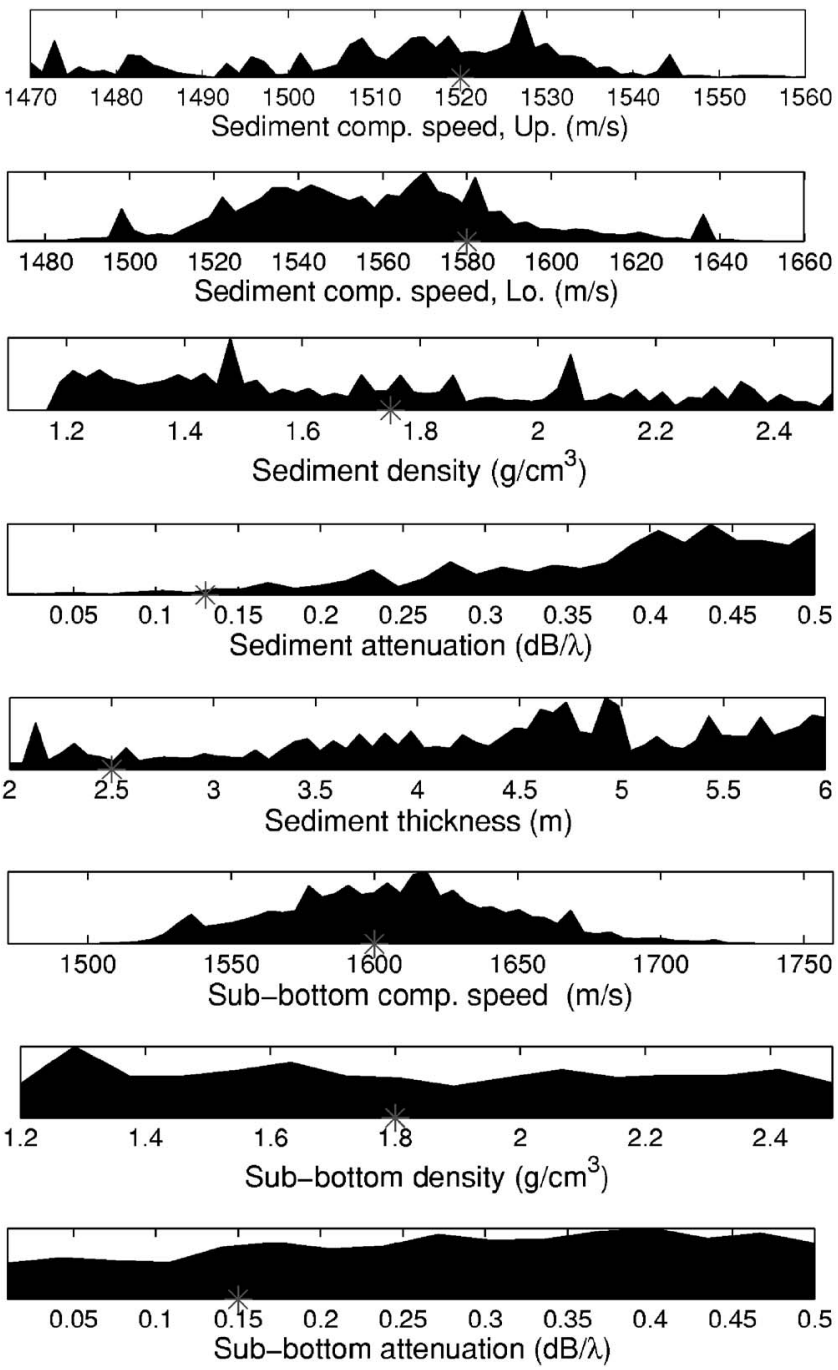

FIG. 12. A posteriori probability distributions for the seafloor parameters based on the last generation of the GA. Only inversions validated by means of source localization during the $A 2$ period are considered. The gray asterisk indicates the baseline value of the parameter.

clearly contribute to improve the convergence of the genetic algorithm to the true model parameters, therefore reducing the inherent uncertainty.

The inversion results with the experimental data are consistent with those obtained with the synthetic data in terms of processor performance comparison. The inversion algorithm included a source localization step with large search bounds aimed at discarding model estimates upon wrong localization result. As no concurrent environmental ground truth data were available the processor performance was evaluated by means of the rate of correct source localization. Based on that criteria, the MUSIC processor achieved the best overall performance. Note that this processor was applied assuming that the dimension of the signal subspace was one, since a coherence optimization step was performed before. This contributed to avoiding a major difficulty with this processorthe estimation of the signal subspace dimension. The minimum-variance processor achieved an exceptional performance in an emission interval where a larger number of signal realizations were available. With this data set successful source localization results were obtained for ranges up to $9 \mathrm{~km}$. 
The EOF coefficients, used to parametrize the water column, were estimated with some uncertainty, although well restricted to subintervals over some periods. A single estimate of the seafloor properties was obtained by means of the maximization of the a posteriori distributions based on the last populations of the genetic algorithm. This result is essentially in line with the baseline model values for compressional speeds in the sediment and subbottom, and density in the sediment. The estimate of the sediment thickness is also well determined. Finally, a strong relation between uncertainty and source localization rate was observed (Fig. 12).

The dimension of the search space appears to be a major impairment for consistently obtaining valid model estimates (by means of correct source localization) over time since, in principle, this cannot be attributed to model mismatch or additive noise.

\section{ACKNOWLEDGMENTS}

The authors would like to thank the NATO Undersea Research Centre for the organization of the MREA'03 sea trial. This work was financed by FCT, Portugal, under fellowship SFRH/BD/12656/2003 and NUACE project, Contract No. POSI/CPS/47824/2002, and the development of the AOB prototype was funded by the Portuguese Ministery of Defense under the LOCAPASS project.

${ }^{1}$ A. Tolstoy, Matched Field Processing for Underwater Acoustics (World Scientific, Singapore, 1993).

${ }^{2}$ A. B. Baggeroer, W. A. Kuperman, and P. N. Mikhalevsky, "An overview of matched field methods in ocean acoustics," IEEE J. Ocean. Eng. 18, 401-424 (1993).

${ }^{3}$ H. P. Bucker, "Use of calculated sound fields and matched-detection to locate sound source in shallow water," J. Acoust. Soc. Am. 59, 368-373 (1976).

${ }^{4}$ A. Tolstoy, O. Diachok, and N. L. Frazer, "Acoustic tomography via matched field processing," J. Acoust. Soc. Am. 89, 1119-1127 (1991).

${ }^{5}$ M. D. Collins, W. A. Kuperman, and H. Schmidt, "Nonliear inversion for ocean-bottom properties," J. Acoust. Soc. Am. 92, 2770-2783 (1992).

${ }^{6} \mathrm{P}$. Gerstoft, "Inversion of acoustic data using a combination of genetic algorithms and the Gauss-Newton approach," J. Acoust. Soc. Am. 97, 2181-2190 (1995)

${ }^{7}$ S. E. Dosso, M. L. Yeremy, J. M. Ozard, and N. R. Chapman, "Estimation of ocean-bottom properties by matched-field inversion of acoustic field data," IEEE J. Ocean. Eng. OE-18, 232-239 (1993).

${ }^{8}$ C. E. Lindsay and N. R. Chapman, "Estimation of ocean-bottom properties by matched-field inversion of acoustic field data," IEEE J. Ocean. Eng. OE-18, 224-231 (1993).

${ }^{9}$ M. D. Collins and W. A. Kuperman, "Focalization: Environmental focusing and source localization," J. Acoust. Soc. Am. 90, 1410-1422 (1991).

${ }^{10}$ D. F. Gingras and P. Gerstoft, "Inversion for geometric parameters in shallow water: Experimental results," J. Acoust. Soc. Am. 97, 3589-3598 (1995).

${ }^{11}$ P. Gerstoft and D. Gingras, "Parameter estimation using multi-frequency range dependent acoustic data in shallow water," J. Acoust. Soc. Am. 99, 2839-2850 (1996).

${ }^{12}$ C. Soares, M. Siderius, and S. M. Jesus, "Source localization in a timevarying ocean waveguide,” J. Acoust. Soc. Am. 112, 1879-1889 (2002).

${ }^{13}$ M. Snellen, D. G. Simons, M. Siderius, J. Sellschopp, and P. L. Nielsen, "An evaluation of the accuracy of shallow water matched field inversion results," J. Acoust. Soc. Am. 109, 514-527 (2001).

${ }^{14}$ R. M. Hamson and R. M. Heitmeyer, "An analytical study of the effects of environmental and system parameters on source localization in shallow water by matched-field processing of a vertical array," J. Acoust. Soc. Am. 86, 1950-1959 (1989).

${ }^{15}$ A. B. Baggeroer, W. A. Kuperman, and H. Schmidt, "Matched field processing: Source localization in correlated noise as an optimum parameter estimation problem," J. Acoust. Soc. Am. 80, 571-587 (1998).

${ }^{16} \mathrm{~A}$. Tolstoy, "Computational aspects of matched field processing in underwater acoustics," in Computational Acoustics, edited by D. Lee, A. Cakmak, and R. Vichnevetsky (North-Holland, Amsterdam, 1990), Vol. 3, pp. 303-310.

${ }^{17}$ Z.-H. Michalopoulou, "Matched-field processing for broad-band source localization,” IEEE J. Ocean. Eng. 21, 384-392 (1996).

${ }^{18}$ Z.-H. Michalopoulou, "Source tracking in the Hudson Canyon experiment," J. Comput. Acoust. 4, 371-383 (1996).

${ }^{19}$ G. J. Orris, M. Nicholas, and J. S. Perkins, "The matched-phase coherent multi-frequency matched field processor," J. Acoust. Soc. Am. 107, $2563-$ 2575 (2000).

${ }^{20}$ C. Soares and S. M. Jesus, "Broadband matched field processing: Coherent and incoherent approaches," J. Acoust. Soc. Am. 113, 2587-2598 (2003).

${ }^{21}$ A. Silva, F. Zabel, and C. Martins, "Acoustic oceanographic buoy: A telemetry system that meets rapid environmental assessment requirements," Sea Technol. 47, 15-20 (2006).

${ }^{22}$ S. M. Jessus, C. Soares, E. Coelho, and P. Picco, "An experimental demonstration of blind ocean acoustic tomography," J. Acoust. Soc. Am. 3, 1420-1431 (2006).

${ }^{23}$ J. Capon, "High-resolution frequency-wavenumber spectrum analysis," Proc. IEEE 57, 1408-1418 (1969).

${ }^{24}$ R. O. Schmidt, "A signal subspace approach to multiple emitter location and spectral estimation," Ph.D. dissertation, Stanford University, Stanford, CA, 1982.

${ }^{25}$ K. Hsu and A. B. Baggeroer, "Application of the maximum-likelihood method (mlm) for sonic velocity logging," Geophysics 51, 780-787 (1986).

${ }^{26} \mathrm{P}$. C. Mignerey and S. Finette, "Multichannel deconvolution of an acoustic transient in an oceanic waveguide," J. Acoust. Soc. Am. 92, 351-364 (1992).

${ }^{27}$ S. Finette, P. C. Mignerey, J. F. Smith, and C. D. Richmond, "Broadband source signature extraction using a vertical array," J. Acoust. Soc. Am. 94, 309-318 (1993).

${ }^{28}$ J. F. Boehme, Advances in Spectrum Analysis and Array Processing, (Prentice Hall, Englewood Cliffs, NJ, 1991), Vol. 2, Chap. 1, pp. 1-63.

${ }^{29}$ C. M. Ferla, M. B. Porter, and F. B. Jensen, "C-SNAP: Coupled SACLANTCEN normal mode propagation loss model," Memorandum SM-274, SACLANTCEN Undersea Research Center, La Spezia, Italy, 1993.

${ }^{30}$ G. Schwartz, "Estimating the dimension of a model," Ann. Stat. 6, 461464 (1978).

${ }^{31}$ J. Rissanen, "Modeling by shortest data description," Automatica 14, 465471 (1978).

${ }^{32} \mathrm{M}$. Wax and T. Kailath, "Detection of signals by information theoretic criteria," IEEE Trans. Acoust., Speech, Signal Process. ASSP-33, 387-392 (1985).

${ }^{33}$ S. Jesus, A. Silva, and C. Soares, "Acoustic Oceanographic Buoy test during the MREA'03 sea trial," Internal Rep. 04/03, SiPLAB/CINTAL, Universidade do Algarve, Faro, Portugal, November 2003.

${ }^{34} \mathrm{~F}$. B. Jensen, "Comparison of transmission loss data for different shallowwater areas with theoretical results provided by a three-fluid normal-mode propagation mode," in Sound Propagation in Shallow Water, edited by O. F. Hastrup and O. V. Oleson (SACLANT Undersea Research Centre, La Spezia, Italy, 1974), Vol. II, pp. 79-92, SACLANTCEN document CP-14.

${ }^{35}$ T. Fassbender, "Erweiterte genetische algorithmen zur globalen optimierung multi-modaler funktionen (Extended genetic algorithms for global optimization of multi-modal functions)." Diplomarbeit, Ruhr-Universität, Bochum, 1995.

${ }^{36}$ C. Soares, S. M. Jesus, and E. Coelho, "Acoustic oceanographic buoy testing during the maritime rapid environmental assessment 2003 sea trial," in Proceedings of the European Conference on Underwater Acoustics 2004, edited by D. Simons, Delft, The Netherlands, pp. 271-279. 